\title{
Mid-infrared Suspended Waveguide Platform and Building Blocks
}

\author{
A. Sánchez-Postigo ${ }^{1, *}$, J. G. Wangüemert-Pérez ${ }^{1}$, J. Soler Penadés ${ }^{2}$, A. Ortega-Moñux ${ }^{1}$, M. \\ Nedeljkovic ${ }^{2}$, R. Halir ${ }^{1,3}$, F. El Mokhtari Mimun', Y.X. Cheng' ${ }^{2}$, Z. Qu², A.Z. Khokhar ${ }^{2}$, A. Osman², W. \\ $\mathrm{Cao}^{2}$, C.G. Littlejohns ${ }^{2}$, P. Cheben ${ }^{4}$, G. Z. Mashanovich², Í. Molina-Fernández ${ }^{1,3}$ \\ 1 Universidad de Málaga, Dpto. Ingeniería de Comunicaciones, ETSI-Telecomunicación, 29071 Málaga, Spain \\ 2 Optoelectronics Research Centre, University of Southampton, Southampton, SO17 1BJ, United Kingdom \\ 3 Bionand Center for Nanomedicine and Biotechnology, Parque Tecnológico de Andalucía, 29590 Málaga, Spain \\ 4 National Research Council Canada, Building M-50, Ottawa, K1A 0R6 Canada \\ *asp@ic.uma.es
}

\begin{abstract}
In this work we present our recent progress in the development of a platform for the mid-infrared wavelength range, based on suspended silicon waveguides with subwavelength metamaterial cladding. The platform has some intrinsic advantages, which make it a very promising candidate for sensing applications in the molecular fingerprint region. Specifically, it can cover the full transparency window of silicon (up to a wavelength of $8 \mu \mathrm{m}$ ), only requires one lithographic etch-step and can be designed for strong light-matter interaction. Design rules, practical aspects of the fabrication process and experimental results of a complete set of elemental building blocks operating at two very different wavelengths, $3.8 \mu \mathrm{m}$ and $7.7 \mu \mathrm{m}$, are discussed. Propagation losses as low as $0.82 \mathrm{~dB} / \mathrm{cm}$ at $\lambda_{0}=3.8 \mu \mathrm{m}$ and $3.1 \mathrm{~dB} / \mathrm{cm}$ at $\lambda_{0}$ $=7.7 \mu \mathrm{m}$ are attained for the interconnecting waveguides.
\end{abstract}

\section{Introduction}

The Mid-InfraRed (MIR, wavelengths from around $2 \mu \mathrm{m}$ to $20 \mu \mathrm{m}$ ) is an interesting wavelength range because of its applications in free-space communications and sensing [1]. This band includes the molecular fingerprint spectral region of many substances [2]. Each molecule is uniquely identified by a specific absorption spectrum which can be measured to determine the presence and concentration of substances as diverse as greenhouse gases, explosives, food, petroleum byproducts, polluting substances, plastics or even human tissues, among others. Regardless of the final application, to operate with a high degree of integration it is necessary to provide the most appropriate platform for this new band of wavelengths. The silicon on insulator (SOI) platform [3], which is widely used for optical communications (around $1.55 \mu \mathrm{m}$ ), could only be used up to a wavelength of $4 \mu \mathrm{m}$, given the strong absorption of the buried silicon dioxide at longer wavelengths. Therefore, to move towards longer wavelengths and cover the full range of the MIR band, it is necessary to develop new platforms. The different alternatives that are currently being considered in the literature can be grouped into three types: i) platforms that combine materials with a greater range of transparency, including silicon on sapphire [4], silicon on silicon nitride [5], chalcogenide crystals [6], germanium on silicon nitride [7], germanium on silicon-on-insulator [8] or germanium on silicon [9] (a summary of the attainable propagation loss of most of these platforms can be found in [2]); ii) air-clad silicon pedestal structures [10]; and iii) suspended platforms that are based on the elimination of the buried oxide layer (BOX), producing suspended silicon waveguides [11-14]. In this way, the complete transparency of silicon, up to $8 \mu \mathrm{m}$, could be covered, while still benefiting from the consolidated SOI manufacturing processes.
Several methods have been proposed to suspend the waveguides. In [11], an array of holes far away from the waveguide core is used to let hydrofluoric (HF) acid reach and remove the BOX, yielding silicon membrane waveguides. Narrower, more stable suspended waveguides are demonstrated in [12], where silicon membranes are fusionbonded to a silicon substrate with pre-patterned air trenches. Alternatively, we have proposed an approach based on periodic subwavelength metamaterial claddings [13, 14]. In these waveguides the holes surrounding the core not only allow the flow of the etching agent used for BOX removal but also enhance the light-matter interaction needed for sensing applications. In this work we will only focus on this latter platform, reviewing the current state of development. The operation principle of the elemental suspended waveguide, practical issues and experimental results of essential building blocks operating at two different wavelengths, $3.8 \mu \mathrm{m}$ and 7.7 $\mu \mathrm{m}$, will be covered throughout the present work. In section 2 the theoretical design of the proposed platform is presented. Then, details of the fabrication process are explained in section 3. Experimental results of the so-far measured devices are provided in section 4 . Finally, in section 5 conclusions are drawn.

\section{Design of the suspended silicon platform}

The complete silicon suspended platform, composed of straight waveguides, $90^{\circ}$ bends and S-bends, multimode interference (MMI) couplers and grating couplers, is designed for the TE polarisation. In our simulations, we employed our in-house 2D Fourier eigenmode expansion tool (FEXEN) [15] together with Rsoft [16] and Photon Design [17] suites to refine dimensions.

\subsection{Interconnecting waveguides}




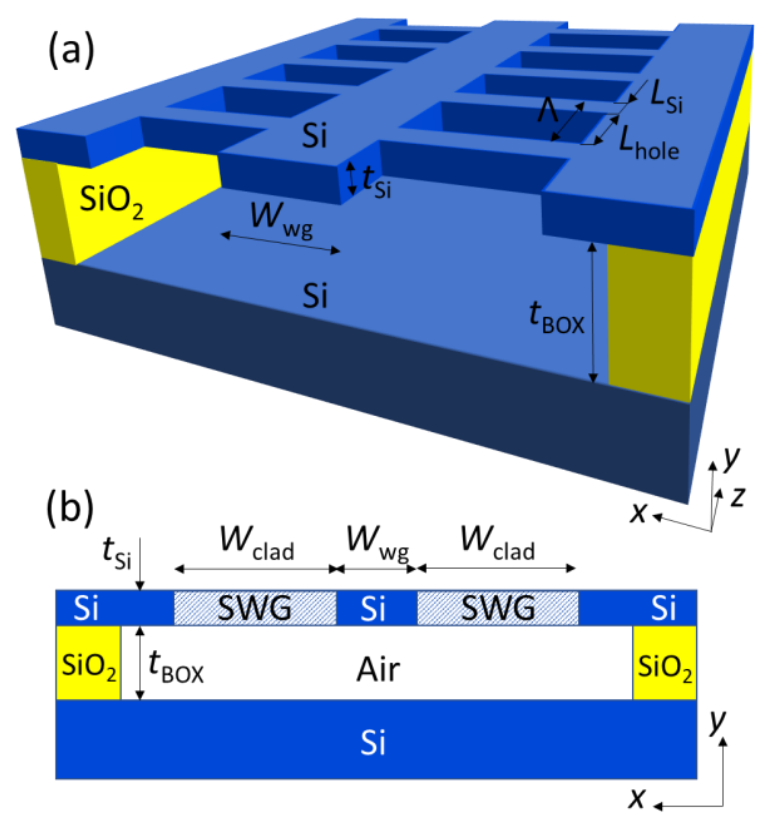

Fig. 1. (a) 3D schematic of the suspended silicon waveguide, (b) $2 D$ cross view.

A schematic representation of the suspended silicon waveguide is shown in Fig. 1(a). As can be seen, it is composed of a silicon core of width $W_{\mathrm{wg}}$ and thickness $t_{\mathrm{Si}}$ which is anchored to the unsuspended lateral silicon areas making use of a subwavelength grating (SWG) [18] pattern of silicon bars of length $L_{\mathrm{Si}}$ with a separation $L_{\text {hole }}$. The periodic cladding of width $W_{\text {clad }}$ represents an equivalent homogeneous medium, by means of which it is possible to control the lateral refractive index contrast required for optical waveguiding (see Fig. 1(b)). The SWG cladding must also perform two additional crucial functions: to have enough mechanical stiffness to support the weight of the wider and hence heavier waveguides present in the circuit and to allow the flow of the HF acid through the holes to remove the buried oxide layer. This makes the design process to determine the adequate dimensions more intricate than that of conventional photonic waveguides, because each one of the aforementioned functions imposes its own constraints.

First, to enable waveguiding, the cladding period $\Lambda=L_{\mathrm{Si}}$ $+L_{\text {hole }}$ must be smaller than the Bragg period [18]. Using our in-house simulator, we estimated $\Lambda_{\text {Bragg }} \approx 600 \mathrm{~nm}\left(\lambda_{0}=3.8\right.$ $\mu \mathrm{m})$ and $1300 \mathrm{~nm}\left(\lambda_{0}=7.7 \mu \mathrm{m}\right)$. Moreover, power leakage to the silicon substrate must be avoided for the fundamental mode by choosing high $t_{\mathrm{Si}}$ and $t_{\mathrm{BOX}}$ values, albeit supported high-order modes should be leaked with small enough $t_{\mathrm{Si}}$ and $t_{\mathrm{BOX}}$ thicknesses. Similarly, power leakage to the lateral unsuspended silicon must also be minimized for the fundamental mode and maximized for high-order modes (if supported). For a given hole size, lateral leakage minimization can be achieved by increasing $W_{\text {clad }}, W_{\text {wg }}$ and the index contrast $\Delta n$ between the waveguide core and the cladding (i.e. by reducing the ratio $L_{\mathrm{Si}} / \Lambda$ ). On the contrary, high-order modes suppression requires reduced $W_{\text {clad, }} W_{\text {wg }}$ and $\Delta n$ values. The influence of the widths $W_{\text {wg }}$ and $W_{\text {clad }}$ on the propagating modes is exemplified in Fig. 2, where a $y$-cut of the two first mode profiles at the centre of the waveguide is represented for several $W_{\mathrm{wg}}$ widths when $W_{\text {clad }}=3 \mu \mathrm{m}$ and $\Delta n=1.6$. In Fig. 2(a), narrowest waveguide, only the fundamental mode is guided, but at the expense of an unacceptable leakage loss. Conversely, in Fig. 2(c), widest waveguide, the fundamental mode is strongly guided, but also the high-order mode is. Figure 2(b) represents an intermediate scenario with a well confined fundamental mode and weakly guided second-order mode. From the figure, it is clear that higher $W_{\text {clad }}$ values will move the lateral unsuspended silicon further from the waveguide core and so leakage will be reduced for both the fundamental and the high-order modes. Increasing $\Delta n$ would have a similar effect by confining the modes within the waveguide core.

Secondly, mechanical robustness requires torque reduction that can be achieved by using small $W_{\text {clad }}$ widths, lowering the weight of the waveguides (small $W_{\mathrm{wg}}$ and $t_{\mathrm{Si}}$ ) and assuring that the silicon strips in the cladding do not break (high $L_{\mathrm{Si}}$ )

Finally, the size of the cladding holes, defined by $W_{\text {clad }}$ and $L_{\text {hole }}$, must be enough to facilitate the flow of HF acid through them.

As it can be seen, the optimum values for each case move towards opposite directions. Therefore, a trade-off solution must be chosen to simultaneously fulfil waveguiding, mechanical stability and HF flow conditions. The final selected values of the interconnecting waveguides at the two operating wavelengths, $3.8 \mu \mathrm{m}$ and $7.7 \mu \mathrm{m}$, are indicated in Table 1. For these parameters, Fig. 3 shows the distribution of the fundamental TE mode supported by each waveguide. For both waveguides, the second-order mode (not represented in the figure) is weakly guided, with leakage loss greater than $50 \mathrm{~dB} / \mathrm{cm}$
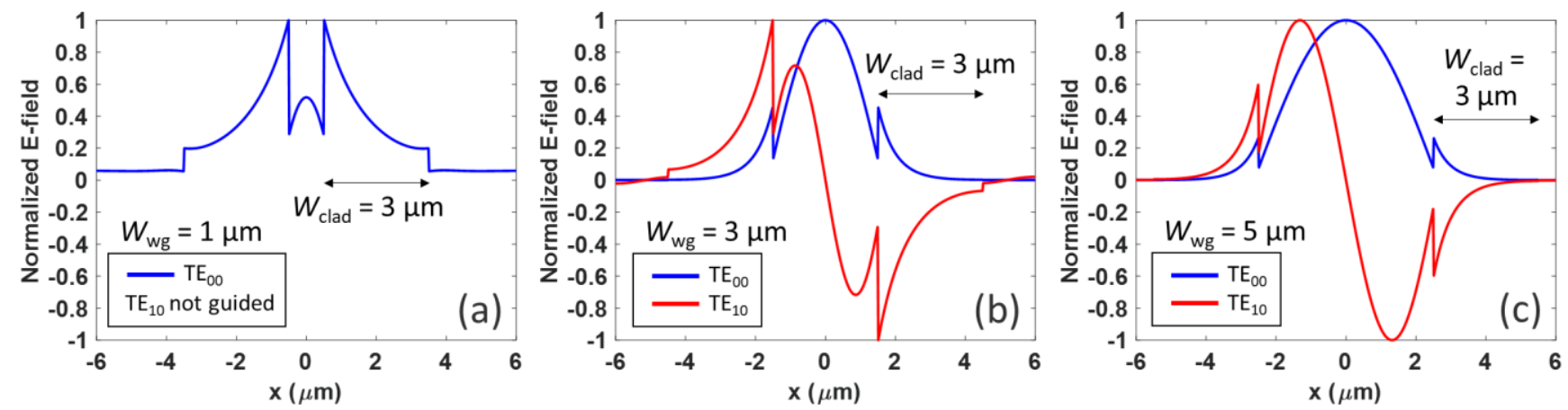

Fig. 2. Cut at the middle of the waveguide core of the first two guided TE mode profiles (electric field normalized to unit peak) for several widths: (a) $W_{w g}=1 \mu \mathrm{m},(\mathrm{b}) W_{w g}=3 \mu \mathrm{m}$ and (c) $W_{w g}=5 \mu \mathrm{m}$. Rest of parameters: $\lambda_{0}=7.7 \mu \mathrm{m}, t_{S i}=1.4$ $\mu \mathrm{m}, W_{\text {clad }}=3 \mu \mathrm{m}$ and $\Delta n=n_{\text {core }}-n_{\text {clad }}=1.6$, where $n_{\text {core }}=3.42$ is the refractive index of Si and $n_{\text {clad }}=1.82$ is the equivalent refractive index of the $S W G$ cladding. 

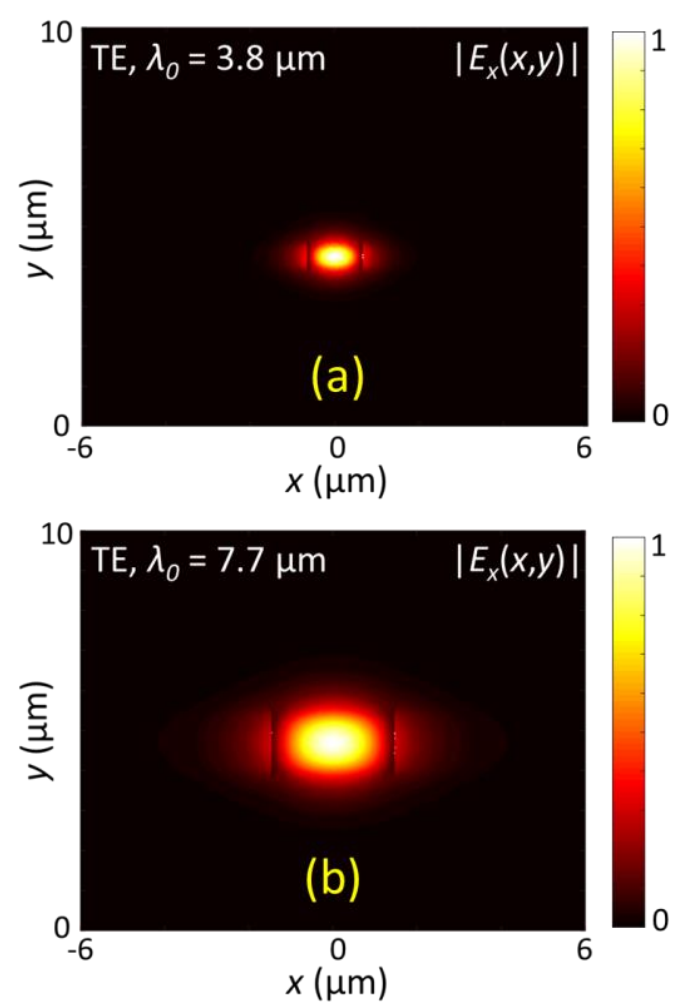

Fig. 3. Magnitude of the main component $\left(E_{x}\right)$ of the simulated TE mode for the designed waveguides at (a) $\lambda_{0}=$ $3.8 \mu \mathrm{m}$ and $(\boldsymbol{b}) \lambda_{0}=7.7 \mu \mathrm{m}$.

Table 1 Designed values of the interconnecting waveguides at both operating wavelengths, $\lambda_{0}=3.8 \mu \mathrm{m}$ and $7.7 \mu \mathrm{m}$

\begin{tabular}{lcc}
\hline Parameter & $\lambda_{0}=3.8 \mu \mathrm{m}$ & $\lambda_{0}=7.7 \mu \mathrm{m}$ \\
& & \\
$\mathrm{W}_{\text {clad }}$ & $2.5 \mu \mathrm{m}$ & $3 \mu \mathrm{m}$ \\
$\mathrm{W}_{\mathrm{wg}}$ & $1.3 \mu \mathrm{m}$ & $2.9 \mu \mathrm{m}$ \\
$t_{\mathrm{Si}}$ & $0.5 \mu \mathrm{m}$ & $1.4 \mu \mathrm{m}$ \\
$t_{\mathrm{BOX}}$ & $3 \mu \mathrm{m}$ & $3 \mu \mathrm{m}$ \\
$L_{\mathrm{Si}}$ & $0.1 \mu \mathrm{m}$ & $0.25 \mu \mathrm{m}$ \\
$L_{\text {hole }}$ & $0.45 \mu \mathrm{m}$ & $0.9 \mu \mathrm{m}$
\end{tabular}

The $L_{\mathrm{Si}}$ value for $\lambda_{0}=3.8 \mu \mathrm{m}$ reflects the main limitation of these waveguides from the fabrication point of view. If the silicon strips become too thin, the suspended waveguide may collapse. Thus, fabrication errors greater than $\Delta L_{\mathrm{Si}}= \pm 50 \mathrm{~nm}$ are unacceptable for the waveguides designed at $\lambda_{0}=3.8 \mu \mathrm{m}$. At longer wavelengths fabrication tolerances are more relaxed because structures are wider and thicker. For our designs, simulations show that errors of this magnitude will slightly affect $\Delta n$ and so the leakage loss should only scarcely change.

Although these waveguides were specifically designed and demonstrated for TE operation, we simulated their behaviour for TM polarisation. At $\lambda_{0}=3.8 \mu \mathrm{m}$ the leakage loss to the substrate and the lateral silicon is so high that waveguiding is not practicable. At $\lambda_{0}=7.7 \mu \mathrm{m}$, on the other hand, the fundamental TM mode has a total loss, including material and leakage loss, less than $1 \mathrm{~dB} / \mathrm{cm}$ higher than the loss for the TE polarisation reported in [14]. A comparative analysis of the sensing capabilities of suspended waveguides, together with a study of leakage loss for both polarisations, can be found in Ref. [19].

\subsection{Bends}

More complex interconnecting waveguides include $90^{\circ}$ bends and S-bends to carry light to other parts of optical circuits. Scanning electron microscope (SEM) images of both suspended structures are shown in Fig. 4. The constant curvature radius $R$ of $90^{\circ}$ bends must be designed to achieve negligible bend loss. Likewise, for sinusoidal S-bends the key parameters are the offset $S$ between the input and the output sections and the length $L$ of the curve. Table 2 shows the final values at $\lambda_{0}=3.8 \mu \mathrm{m}$ and $7.7 \mu \mathrm{m}$.

Table 2 Designed values of the $90^{\circ}$ bends and S-bends at both operating wavelengths, $\lambda_{0}=3.8 \mu \mathrm{m}$ and $7.7 \mu \mathrm{m}$

\begin{tabular}{lcc}
\hline Parameter & $\lambda_{0}=3.8 \mu \mathrm{m}$ & $\lambda_{0}=7.7 \mu \mathrm{m}$ \\
\hline$R$ & $16 \mu \mathrm{m}$ & $35 \mu \mathrm{m}$ \\
$S$ & $7 \mu \mathrm{m}$ & $5 \mu \mathrm{m}$ \\
$L$ & $41 \mu \mathrm{m}$ & $75 \mu \mathrm{m}$
\end{tabular}

\subsection{MMI couplers}

Multimode interference (MMI) couplers are essential building blocks in integrated photonics, as they can be used as power splitters and combiners. These devices comprise a wide multimode waveguide of width $W_{\mathrm{MMI}}$ and length $L_{\mathrm{MMI}}$ with $M$ input and $N$ output waveguides of width $W_{\mathrm{a}}$ and separation $W_{s}$. Figure 5 shows a schematic of a suspended $2 \times 2$ MMI $(M=2, N=2)$. The working principle is based on the self-imaging phenomenon: when light is injected into the wide waveguide through one of the access ports, labelled from 1 to 4 in the figure, the interference between the supported modes produces several replicas or images of the input mode at different positions along the propagation direction. A further explanation of MMI operation and design procedures can be found in [20].

In this work we have designed $2 \times 2$ MMIs for both operating wavelengths. These couplers typically exhibit more compactness, bandwidth and relaxed fabrication tolerances than conventional directional couplers. The performance metrics of MMIs are the excess loss (1), the power imbalance (2) and the phase error (3), which can be calculated as

$$
\begin{gathered}
E L=-10 \log _{10}\left(\left|S_{31}\right|^{2}+\left|S_{41}\right|^{2}\right), \\
I B=-10 \log _{10}\left(\left|S_{31}\right|^{2} /\left|S_{41}\right|^{2}\right), \\
P E=\angle\left(S_{31} / S_{41}\right)-90^{\circ},(3)
\end{gathered}
$$
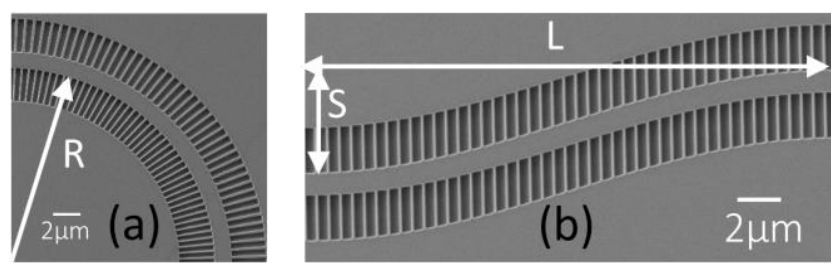

Fig. 4. (a) SEM image of suspended $90^{\circ}$ bend with constant radius $\mathrm{R}$, (b) SEM image of suspended S-bend with sinusoidal shape defined by length $\mathrm{L}$ and offset $\mathrm{S}$. 


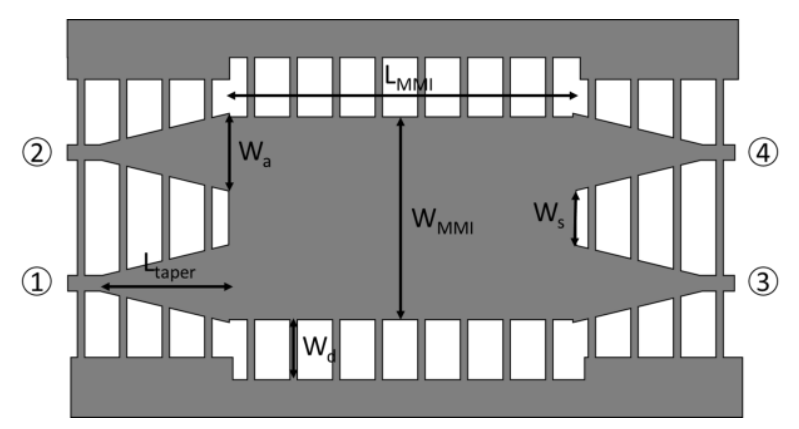

Fig. 5. Schematics of a suspended $2 \times 2$ MMI. Circled numbers designate each access port.

where $S_{\mathrm{ji}}$ is the s-parameter associated to the input port $i$ and the output port $j$.

Table 3 summarizes the geometrical parameters of the designed MMIs. Likewise, Fig. 6 shows the simulated performance of both devices with respect to the wavelength. It can be seen that $E L<0.4 \mathrm{~dB},|I B|<0.3 \mathrm{~dB}$ and $|P E|<1.5^{\circ}$ in all cases for the depicted ranges.

Table 3 Designed values of the $2 \times 2$ MMI couplers at both operating wavelengths, $\lambda_{0}=3.8 \mu \mathrm{m}$ and $7.7 \mu \mathrm{m}$

$\begin{array}{lcc}\text { Parameter } & \lambda_{0}=3.8 \mu \mathrm{m} & \lambda_{0}=7.7 \mu \mathrm{m} \\ W_{a} & 3.1 \mu \mathrm{m} & \\ W_{s} & 1.3 \mu \mathrm{m} & 1.84 \mu \mathrm{m} \\ W_{d} & 2 \mu \mathrm{m} & 2 \mu \mathrm{m} \\ W_{M M I} & 7.1 \mu \mathrm{m} & 13.38 \mu \mathrm{m} \\ L_{M M I} & 71.5 \mu \mathrm{m} & 136.9 \mu \mathrm{m} \\ L_{\text {taper }} & 40 \mu \mathrm{m} & 30 \mu \mathrm{m}\end{array}$

\subsection{Grating couplers}

Surface grating couplers are periodic waveguides employed to couple light between an optical chip and an optical fibre (see Fig. 7(a)). Unlike edge couplers, surface grating couplers allow wafer scale testing and avoid the necessity of polishing or cleaving the chip facet [21]. This latter advantage is especially appropriate for suspended structures, which can easily collapse in polishing/cleaving processes. The key parameters that are usually utilized to characterize the performance of these devices are the coupling efficiency, which can be defined as the fraction of

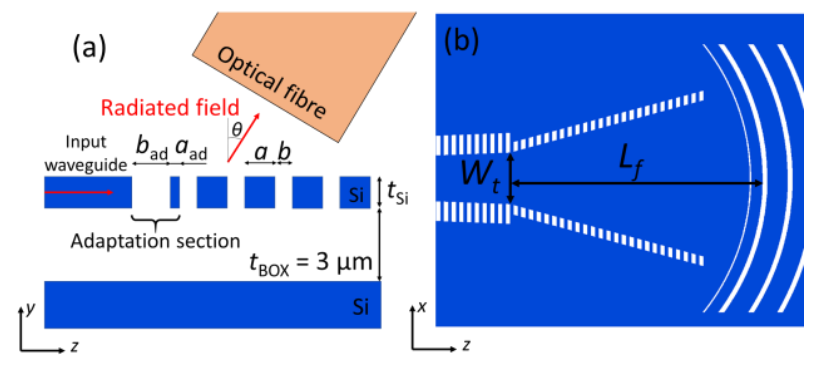

Fig. 7. Schematic (a) side view (including input waveguide and optical fibre) and (b) top view (including input waveguide) of the designed focusing grating coupler at $\lambda_{0}=7.7 \mu \mathrm{m}$.

the input power that is coupled into the optical fibre, and the $1-\mathrm{dB}$ bandwidth.

At $\lambda_{0}=3.8 \mu \mathrm{m}$ we employed grating couplers available in the literature for the suspended membrane platform [11, 22], but we specifically designed new focusing couplers (Fig. 7(b)) for the long-wave range at $\lambda_{0}=7.7 \mu \mathrm{m}$. Table 4 contains the geometrical parameters of this grating coupler. Figure 8 shows the propagation of the electric field radiated by the grating coupler and its calculated coupling efficiency as a function of the wavelength. As it can be seen, a simulated peak coupling efficiency of $58 \%$ and a $1-\mathrm{dB}$ bandwidth of 230 $\mathrm{nm}$ are achieved for a mid-infrared optical fibre with a mode field diameter of $29.52 \mu \mathrm{m}$. This grating coupler radiates with an angle of $\sim 19^{\circ}$. Back-reflections are reduced with an adaptation section composed of a Si strip of length $a_{\text {ad }}$ and a hole of length $b_{\text {ad }}$ before the grating (see Fig. 7(a)).
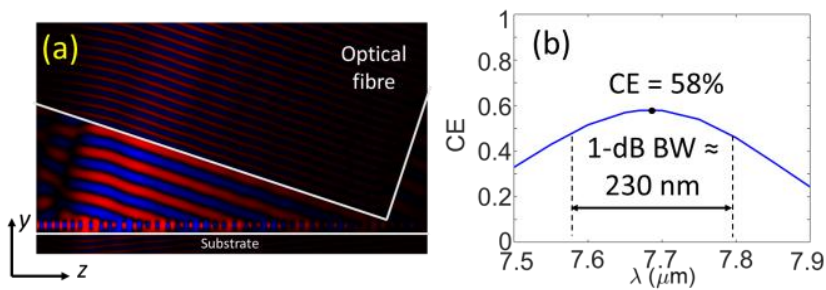

Fig. 8. (a) Radiated electric field of the grating coupler, (b) Simulated coupling efficiency of the designed grating coupler.
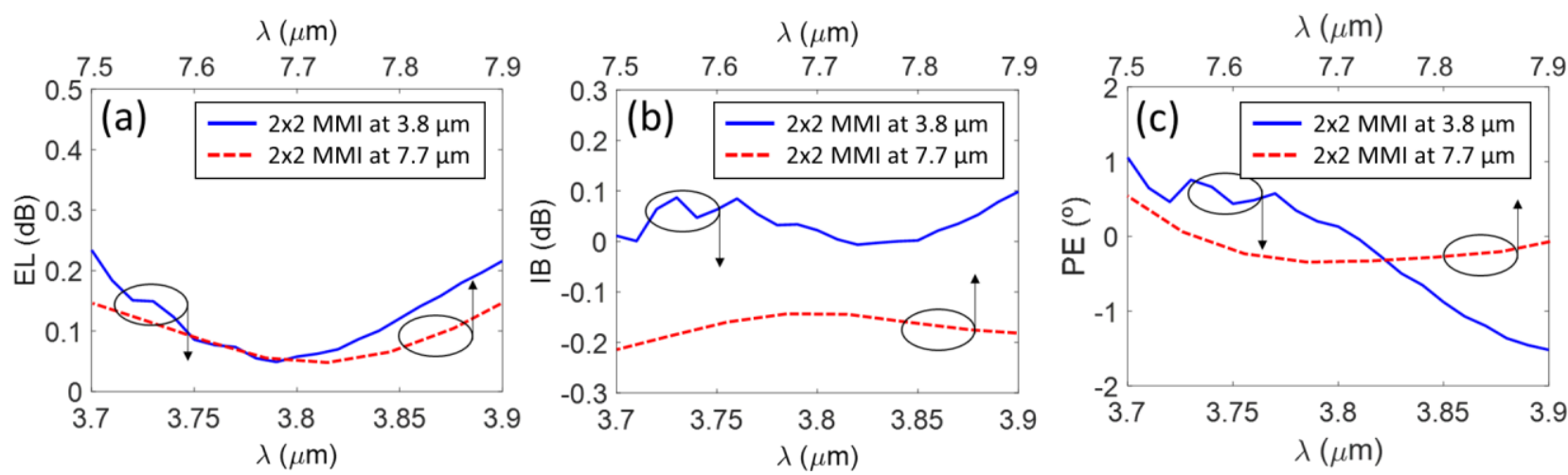

Fig. 6. Simulated (a) excess loss, (b) power imbalance and (c) phase error for the designed $2 x 2$ MMIs at $\lambda_{0}=3.8 \mu \mathrm{m}$ (solid blue line) and $7.7 \mu \mathrm{m}$ (red dashed line). 


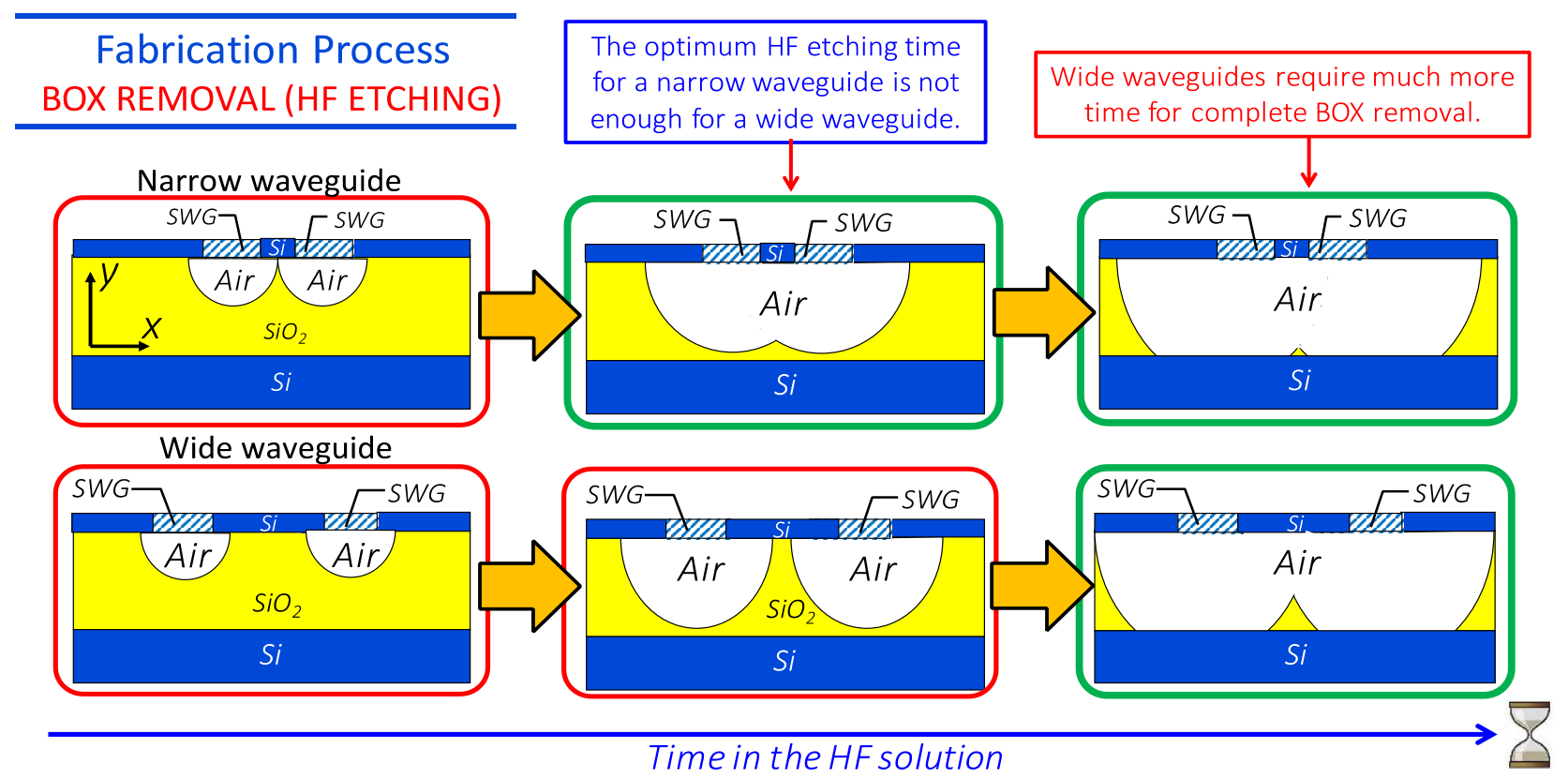

Fig. 9. Graphical illustration of the wet etching process to remove the BOX beneath the devices

Table 4 Selected values of the designed grating coupler at $\lambda_{0}$ $=7.7 \mu \mathrm{m}$

\begin{tabular}{lc}
\hline Parameter & $\lambda_{0}=7.7 \mu \mathrm{m}$ \\
\hline & \\
$a$ & $2.75 \mu \mathrm{m}$ \\
$b$ & $1 \mu \mathrm{m}$ \\
$a_{a d}$ & $1.6 \mu \mathrm{m}$ \\
$b_{a d}$ & $0.5 \mu \mathrm{m}$ \\
$W_{t}$ & $4.5 \mu \mathrm{m}$ \\
$L_{f}$ & $22.5 \mu \mathrm{m}$ \\
$N$ & 40
\end{tabular}

\section{Fabrication}

The fabrication process of any device in the proposed suspended platform requires only one lithographic etch step after the structures have been patterned in a ZEP-520A photoresist. Thus, dry etching with an inductively coupled plasma (ICP) tool is utilized to transfer the pattern to the silicon layer. Then, an additional wet etching is applied to locally remove the buried oxide (BOX) beneath the silicon layer. This consists in dipping the chip into a 1:7 HF bath to remove the silicon dioxide. The difficulty arises from the required immersion time, which, as is shown in Fig. 9, is greater for wide waveguides (e.g. MMIs) than for narrow ones (e.g. interconnecting waveguides). Although the HF acid is highly selective and, in theory, should not affect the silicon, measurements done with an elipsometer show that in practice the silicon is slightly affected, suffering from an overetching which produces a reduction in both the thickness and width of the nominal dimensions (see Fig. 10). This silicon overetching can have dramatic consequences not only in the performance of the device but also in its mechanical stability. To cancel this effect out, holes patterned in the photoresist need to be smaller in such a way that the silicon overetching caused by the HF is compensated. Therefore, required biasing for the dry etch must be accurately known in advance. Figures 11(a) and 11(b) show, respectively, the consequences of the dip time: when it is too short, the BOX is not completely removed, and when it is excessive, the overetching is so large that it ends up breaking the silicon bars. Figure 11(c), in turn, shows the results of a more optimised dip time. At $\lambda_{0}=3.8 \mu \mathrm{m}$, about 50-minute etch was enough to suspend the widest structures, while around 2 hours were required at $\lambda_{0}=7.7 \mu \mathrm{m}$.

\section{Experimental results}

The straight waveguides, bends and MMI couplers designed at $\lambda_{0}=3.8 \mu \mathrm{m}$ have been characterized. At $\lambda_{0}=7.7$ $\mu \mathrm{m}$ only the propagation losses of straight and bent waveguides have been measured. Tables 5 and 6 summarize the attained performance for each structure. Figure 12 shows the normalised measured loss of several waveguides with different lengths and the calculated propagation loss. Note that at $\lambda_{0}=7.7 \mu \mathrm{m}$ silicon has material losses around $2 \mathrm{~dB} / \mathrm{cm}$ [23], what justifies the higher loss of waveguides at this wavelength with respect to their counterparts at $\lambda_{0}=3.8 \mu \mathrm{m}$.

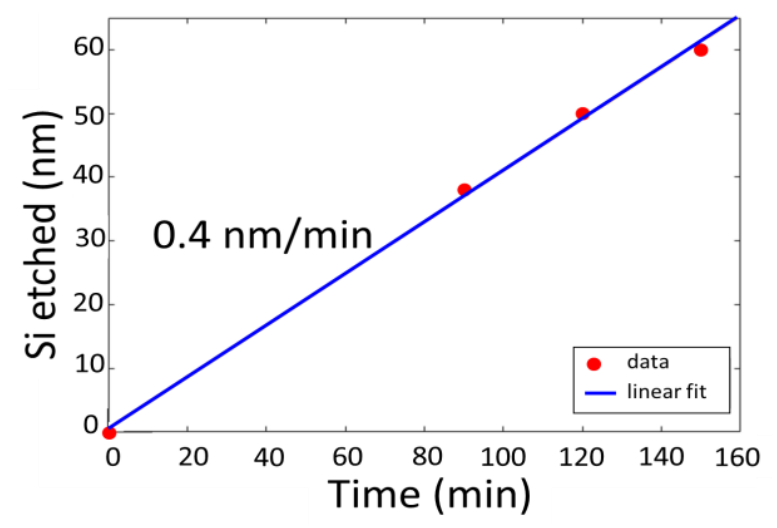

Fig. 10. Undesired reduction of the vertical silicon thickness as a function of the immersion time into the HF.

Measurements were taken with an elipsometer after 0, 90, 120 and 150 min of immersion into the HF. 


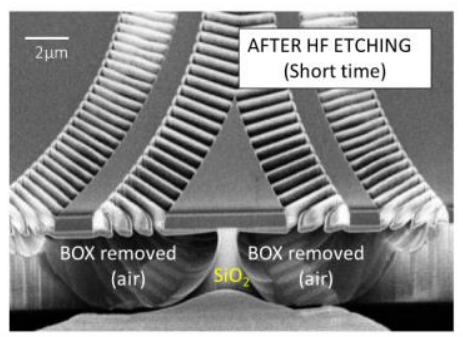

(a)

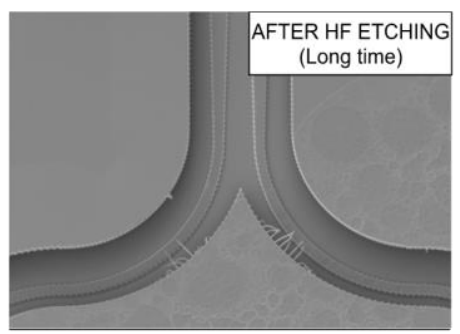

(b)

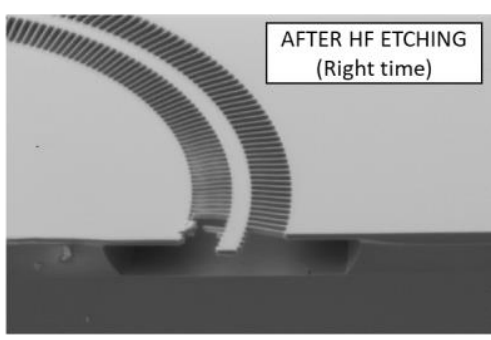

(c)

Fig. 11. SEM images of fabricated interconnecting waveguides when the immersion time is (a) too short, (b) too long and (c) optimized.

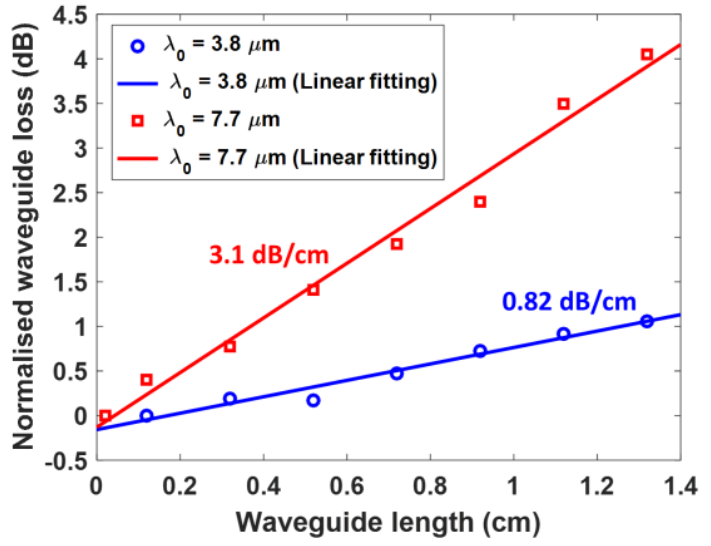

Fig. 12. Measured loss of the waveguides designed for TE polarisation at $\lambda_{0}=3.8 \mu \mathrm{m}$ (blue) and $\lambda_{0}=7.7 \mu \mathrm{m}$ (red) for several lengths. The propagation loss is also indicated.

Table 5 Experimental performance summary of some basic building blocks at $\lambda_{0}=3.8 \mu \mathrm{m}$ (TE polarisation)

Device Parameter

$\begin{array}{lll}\text { Straight waveguide } & \text { Propagation loss } & 0.82 \mathrm{~dB} / \mathrm{cm} \\ \text { S-bends } & \text { Propagation loss } & 0.01 \mathrm{~dB} / \mathrm{bend} \\ 90^{\circ} \text {-bends } & \text { Propagation loss } & 0.02 \mathrm{~dB} / \mathrm{bend} \\ 2 \times 2 \mathrm{MMI} & \text { Excess loss } & <0.3 \mathrm{~dB} \\ & \text { Imbalance } & <0.3 \mathrm{~dB}\end{array}$

Table 6 Experimental performance summary of some basic building blocks at $\lambda_{0}=7.7 \mu \mathrm{m}$ (TE polarisation)

\begin{tabular}{lll}
\hline Device & \multicolumn{1}{c}{ Parameter } \\
\hline & & \\
Straight waveguide & Propagation loss & $3.1 \mathrm{~dB} / \mathrm{cm}$ \\
S-bends & Propagation loss & $0.06 \mathrm{~dB} / \mathrm{bend}$ \\
$90^{\circ}$-bends & Propagation loss & $0.08 \mathrm{~dB} / \mathrm{bend}$ \\
$2 \times 2 \mathrm{MMI}$ & $\begin{array}{l}\text { Experimental characterisation in } \\
\text { progress }\end{array}$
\end{tabular}

\section{Conclusion}

We have presented and reviewed the current state of a recent platform for the mid-infrared band, the suspended silicon waveguide with SWG lateral cladding, which would allow us to extend the usage of silicon photonics up to wavelengths of $8 \mu \mathrm{m}$. The first results at $3.8 \mu \mathrm{m}$ and $7.7 \mu \mathrm{m}$ in waveguides, bends and MMIs are very promising, thus paving the way to a complete set of new devices and sensors and leaving open the possibility of suspending other materials such as silicon nitride or germanium to cover the full midinfrared band.

\section{Acknowledgements}

This work has been supported by the Spanish Ministerio de Economía y Competitividad, Programa Estatal de Investigación, Desarrollo e Innovación Orientada a los Retos de la Sociedad (cofinanciado FEDER), Proyecto TEC201680718-R; the Spanish Ministerio de Educación, Cultura y Deporte (FPU14/06121), the Universidad de Málaga and two EPSRC grants: High Value Photonic Manufacturing (EP/N00762X/1) and CORNERSTONE (EP/L021129/1).

\section{References}

[1] Soref, R.: 'Mid-infrared photonics in silicon and germanium', Nature Phot., 2010, 4, pp. 495-497.

[2] Hu, T., et al.: 'Silicon photonic platforms for midinfrared applications', Photon. Res., 2017, 5, pp. 417-430.

[3] Soref, R.A., et al.: 'Silicon waveguided components for the long-wave infrared region', J. Opt. A: Pure Appl. Opt., 2006, 8, pp. 840-848.

[4] Baehr-Jones, T., et al.: 'Silicon-on-sapphire integrated waveguides for the mid-infrared', Opt. Express, 2010, 18, (12), pp. 12127-12135.

[5] Khan, S., et al.: 'Silicon-on-nitride waveguides for midand near-infrared integrated photonics', Appl. Phys. Lett., 2013, 102, (12), p. 121104.

[6] Han, Z., et al.: 'On-chip mid-infrared gas detection using chalcogenide glass waveguide’, Appl. Phys. Lett., 2016, 108 , p. 141106.

[7] Li, W., et al.: 'Germanium-on-silicon nitride waveguides for mid-infrared integrated photonics', Appl. Phys. Lett., 2016, 109, p. 241101.

[8] Malik, A., et al.: 'Ge-on-Si and Ge-on-SOI thermo-optic phase shifters for the mid-infrared', Opt. Express, 2014, 22, (23), pp. 28479-28488.

[9] Nedeljkovic, M., et al.: 'Surface-grating-coupled lowloss Ge-on-Si rib waveguides and multimode 
interferometers', IEEE Phot. Tech. Lett., 2015, 27, (10), pp. 1040-1043.

[10] Lin, P. T., et al.: 'Air-clad silicon pedestal structures for broadband mid-infrared microphotonics', Opt. Letters, 2013, 38, (7), pp. 1031-1033.

[11] Cheng, Z., et al.: 'Mid-infrared suspended membrane waveguide and ring resonator on silicon-on-insulator', IEEE Photonics J., 2012, 4, (5), pp. 1510-1519.

[12] Chiles, J., et al.: 'High-contrast, all-silicon waveguiding platform for ultra-broadband mid-infrared photonics', Appl. Phys. Lett., 2013, 103, p. 151106.

[13] Soler Penades, J., et al.: 'Suspended silicon midinfrared waveguide devices with subwavelength grating metamaterial cladding', Opt. Express, 2016, 24, pp. 22908 22916.

[14] Soler Penades, J., et al.: 'Suspended silicon waveguides for long-wave infrared wavelengths', Opt. Letters, 2018, 43, pp. 795-798.

[15] Zavargo-Peche, L., et al., 'Fourier based combined techniques to design novel sub-wavelength optical integrated devices', Prog. Electromagnetics Res. 2012, 123, pp. 447-465.

[16] 'Photonic Design Software | Rsoft Products', https://www.synopsys.com/optical-solutions/rsoft.html, accessed 18 June 2018.

[17] 'Photon Design', https://www.photond.com/index.htm, accessed 18 June 2018.

[18] Halir, R., et al.: 'Waveguide sub-wavelength structures: a review of principles and applications', Laser Photonics Rev. 2015, 9, (1), pp. 25-49.

[19] Wangüemert-Pérez, J. G., et al.: 'Subwavelength structures for silicon photonics biosensing', Optics \& Laser Technol., 2019, 109, pp. 437-448.

[20] Soldano, L. B., Pennings, E. C. M.: 'Optical multimode interference devices based on self-imaging: Principles and applications', J. Lightwave Technol., 1995, 13, (4), pp. 615627.

[21] Taillaert, D., et al.: 'Compact efficient broadband grating coupler for silicon-on-insulator waveguides', Opt. Lett., 2004, 29, (23), pp. 2749-2751.

[22] Halir, R., et al.: 'Waveguide grating coupler with subwavelength microstructures', Opt. Express, 2009, 34, (9), pp. 1408-1410.

[23] Chandler-Horowitz, D., Amirtharaj, P. M.: 'Highaccuracy, midinfrared $(450 \mathrm{~cm}-1 \leq \omega \leq 4000 \mathrm{~cm}-1)$ refractive index of silicon', J. Appl. Phys, 2005, 97, p. 123526. 